\title{
Lactobacillus reuteri-derived extracellular vesicles maintain intestinal immune homeostasis against lipopolysaccharide- induced inflammatory responses in broilers
}

\author{
Rujiu Hu, Hua Lin, Mimi Wang, Yuezhen Zhao, Haojing Liu, Yuna Min, Xiaojun Yang, Yupeng Gao \\ and Mingming Yang ${ }^{*}$ (D)
}

\begin{abstract}
Background: Lactobacillus reuteri strains are widely used as probiotics to prevent and treat inflammatory bowel disease by modulating the host's immune system. However, the underlying mechanisms by which they communicate with the host have not been clearly understood. Bacterial extracellular vesicles (EVs) have been considered as important mediators of host-pathogen interactions, but their potential role in commensals-host crosstalk has not been widely studied. Here, we investigated the regulatory actions of EVs produced by L. reuteri BBC3, a gut-associated commensal bacterium of Black-Bone chicken, in the development of lipopolysaccharide (LPS)-induced intestinal inflammation in a chicken model using both in vivo and in vitro experiments.

Results: L. reuteri BBC3 produced nano-scale membrane vesicles with the size range of 60-250 nm. Biochemical and proteomic analyses showed that L. reuteri BBC3-derived EVs (LrEVs) carried DNA, RNA and several bioactive proteins previously described as mediators of other probiotics' beneficial effects such as glucosyltransferase, serine protease and elongation factor Tu. In vivo broiler experiments showed that administration of LrEVs exerted similar effects as $L$. reuteri BBC3 in attenuating LPS-induced inflammation by improving growth performance, reducing mortality and decreasing intestinal injury. LrEVs suppressed the LPS-induced expression of pro-inflammatory genes (TNF- $\alpha, I L-1 \beta, I L-6, I L-17$ and IL-8), and improved the expression of anti-inflammatory genes (IL-10 and TGF- $\beta$ ) in the jejunum. LrEVs could be internalized by chicken macrophages. In vitro pretreatment with LrEVs reduced the gene expression of TNF- $\alpha, I L-1 \beta$ and $I L-6$ by suppressing the NF-KB activity, and enhanced the gene expression of $I L-10$ and TGF- $\beta$ in LPS-activated chicken macrophages. Additionally, LrEVs could inhibit Th1- and Th17-mediated inflammatory responses and enhance the immunoregulatory cells-mediated immunosuppression in splenic lymphocytes of LPS-challenged chickens through the activation of macrophages. Finally, we revealed that the reduced content of both vesicular proteins and nucleic acids attenuated the suppression of LrEVs on LPS-induced inflammatory responses in ex vivo experiments, suggesting that they are essential for the LrEVs-mediated immunoregulation.
\end{abstract}

\footnotetext{
* Correspondence: ymm@nwsuaf.edu.cn

College of Animal Science and Technology, Northwest A\&F University,

Yangling 712100, Shaanxi, China
}

(c) The Author(s). 2021 Open Access This article is licensed under a Creative Commons Attribution 4.0 International License, which permits use, sharing, adaptation, distribution and reproduction in any medium or format, as long as you give appropriate credit to the original author(s) and the source, provide a link to the Creative Commons licence, and indicate if changes were made. The images or other third party material in this article are included in the article's Creative Commons licence, unless indicated otherwise in a credit line to the material. If material is not included in the article's Creative Commons licence and your intended use is not permitted by statutory regulation or exceeds the permitted use, you will need to obtain permission directly from the copyright holder. To view a copy of this licence, visit http://creativecommons.org/licenses/by/4.0/ The Creative Commons Public Domain Dedication waiver (http://creativecommons.org/publicdomain/zero/1.0/) applies to the data made available in this article, unless otherwise stated in a credit line to the data. 
(Continued from previous page)

Conclusions: We revealed that LrEVs participated in maintaining intestinal immune homeostasis against LPSinduced inflammatory responses in a chicken model. Our findings provide mechanistic insight into how commensal and probiotic Lactobacillus species modulate the host's immune system in pathogens-induced inflammation.

Keywords: Chickens, Extracellular vesicles, Immune responses, Inflammation, Lactobacillus, Microbiota-host communication, Probiotics

\section{Background}

Membrane vesicles (MVs) have been recognized as a form of cell-cell communication used by almost all domains of life: bacteria, archaea and eukaryotes [1]. Over the years, they have been largely ignored in the field of microbiology. It is only in the last decade that advances in biochemical analysis have led researchers to begin to elucidate the biogenesis and functions of MVs [2, 3]. Bacterial MVs are released from the cell surface into the extracellular environment, and thus also referred to as extracellular vesicles (EVs). These vesicles are composed of spherical proteolipids with a diameter ranging from 50 to $500 \mathrm{~nm}$ [1]. Bacterial EVs contain various bioactive molecules of the parental bacteria, including proteins, lipids, nucleic acids and polysaccharides, which are involved in a large number of pathological and physiological functions in intercellular interactions such as nutrient acquisition, biofilm formation, stress response, delivery of toxins and virulence factors and invasion of host and immune regulation [2,3]. Gram-negative bacterial EVs are mainly derived from the outer membrane of the cell envelope, and thus also termed outer membrane vesicles (OMVs) [4]. Most of the early work regarding bacterial EVs was conducted mainly on Gramnegative bacteria, especially pathogenic bacteria, which has shown that the EVs can mediate bacterial pathogenesis and invasion by delivering toxins and virulence factors into the host cells [4]. OMVs can also activate the innate and adaptive immune responses and induce the protective immunity similar to that induced by the intact bacteria in vivo, suggesting that these OMVs can be used for the development of novel vaccine candidates and adjuvants $[5,6]$.

Few studies, however, have been performed on Grampositive bacterial EVs, because it is believed to be difficult for Gram-positive bacteria to release EVs due to the presence of a thick cell wall [1]. It was not until 2009 when direct evidence was provided for the existence of EVs in Gram-positive bacteria by transmission electron microscopy and proteomic analyses of EVs from Staphylococcus aureus [7]. Since then, more and more Gram-positive bacteria have been demonstrated to be able to produce EVs, such as Bacillus anthracis [8], Streptococcus pneumoniae [9], Bacillus subtilis [10] and Clostridium perfringens [11]. As the most common
Gram-positive bacteria, several Lactobacillus species have been recently discovered to produce EVs. Rubio et al. [12] demonstrated the probiotic strain $L$. casei BL23 produced biofunctional EVs. Li et al. [13] showed that $L$. plantarum WCFS1 secreted EVs with an average size of $101 \mathrm{~nm}$. EVs derived from L. acidophilus and L. paracasei have also been isolated and characterized [14, 15]. Recently, several studies also demonstrated the existence of EVs in L. reuteri strains [14, 16]. However, the potential roles and functions of these Lactobacillus-derived vesicles have not been extensively studied.

With the gradual ban on the usage of antibiotic growth promoters in today's intensive broiler industry worldwide, probiotics have been widely used as alternatives to prevent and treat various inflammatory disorders in the gastrointestinal tract. L. reuteri species have been demonstrated to have beneficial attributes, particularly the ability to modulating the development and function of the host's immune system, in humans and various animals, including rodents, pigs, turkeys and chickens [17, 18]. A previous study has demonstrated that $L$. reuteri inhibits the secretion of pro-inflammatory cytokines tumour necrosis factor (TNF)- $\alpha$ and interleukin (IL)- 6 in murine dendritic cells (DCs) [19]. Lin et al. [20] revealed that $L$. reuteri exerted the suppressive effect on the production of TNF- $\alpha$ in lipopolysaccharide (LPS)-challenged human macrophages $(\mathrm{M} \phi)$. L. reuteri has also been shown to have an ability to induce the development of regulatory $\mathrm{T}$ cells (Tregs) that inhibit inflammatory responses $[21,22]$. In chickens, $L$. reuteri is one of the most abundant Lactobacillus species in the intestine and has been shown to have an antiinflammatory effect against pathogens [23-25]. However, the underlying molecular mechanisms of $L$. reuteri-host interaction have not been clearly understood.

Based on these findings, the present study was initiated to explore whether EVs derived from the probiotic strain $L$. reuteri $\mathrm{BBC} 3$ (LrEVs), a gut-associated commensal bacterium of Black-Bone chicken, mediate immunoregulation in the chicken intestine, especially antiinflammatory actions. To achieve this aim, we characterized the protein composition of LrEVs using proteomic analysis, evaluated the protective effects of LrEVs in a chicken model of LPS-induced intestinal inflammation and investigated the immune responses mediated by LrEVs in the LPS-activated $M \phi$ model and $M \phi$-splenic 
lymphocytes coculture system in vitro. We further attempted to understand the roles of vesicular proteins and nucleic acids in LrEVs-mediated immunomodulation using an ex vivo experiment.

\section{Materials and methods}

\section{Bacterial strain and culture conditions}

L. reuteri BBC3 (GenBank accession: MT476913) was previously isolated by our laboratory from the gastrointestinal tract of healthy Black-Bone chickens in freerange farms (Lueyang, Shaanxi Province, China). This strain was identified by morphological and physicochemical characterizations and $16 \mathrm{~S}$ rRNA sequence analysis. L. reuteri $\mathrm{BBC} 3$ was routinely incubated in MRS broth at $37^{\circ} \mathrm{C}$ under anaerobic conditions.

\section{Isolation and purification of LrEVs}

LrEVs were isolated from the culture supernatants of $L$. reuteri $\mathrm{BBC} 3$ using a series of (ultra)centrifugation steps as described previously [26, 27]. Briefly, after growing in MRS broth for $16 \mathrm{~h}$, the bacteria-free culture supernatants were collected by centrifugation $(12,000 \times g, 20 \mathrm{~min}$, $4{ }^{\circ} \mathrm{C}$ ), filtered with a $0.45-\mu \mathrm{m}$ bottle top vacuum filter (Corning), and then concentrated using an Amicon Ultrafiltration system (Millipore) with a $100-\mathrm{kDa}$ filter. The LrEVs pellets were obtained by ultracentrifugation $\left(150,000 \times g, 2 \mathrm{~h}, 4{ }^{\circ} \mathrm{C}\right)$, washed in sterile phosphate buffer saline (PBS; pH 7.4) and then purified by density gradient centrifugation. For purification, LrEVs were covered with discontinuous Optiprep (Sigma, \#D1556) stepgradient ranging from $10 \%$ to $55 \%(\mathrm{w} / \mathrm{v})$ and subjected to ultracentrifugation $\left(16 \mathrm{~h}, 180,000 \times g, 4^{\circ} \mathrm{C}\right)$. After centrifugation, nanoparticle tracking analysis (NTA) was performed to detect the particle numbers of the resulting fractions. These vesicle-rich fractions were pooled and ultra-centrifuged to remove OptiPrep. The purified LrVs were subjected to filtration $(0.45 \mu \mathrm{m}$, Millipore) to eliminate the potential bacterial contamination and stored at $-80^{\circ} \mathrm{C}$ until future use.

\section{Electron microscopy and nanoparticle tracking analysis}

Morphological characteristics of $L$. reuteri $\mathrm{BBC} 3$ and LrEVs were detected by scanning electron microscopy using a Field Emission Scanning Electron Microscope (S-4800, Hitachi, Tokyo, Japan). The LrEVs were also viewed by transmission electron microscopy using JEM1011 Electron Microscope at $100 \mathrm{kV}$ (JEOL, Tokyo, Japan) as described previously [28]. NTA was performed to detect the diameter and particle number of the LrEVs using an NS300 nanoparticle analyzer (Malvern, Worchestershire, UK) with the operating parameters as described in our previous study [27].

\section{Biochemical analysis}

The protein content of the LrEVs was determined by the TaKaRa BCA Protein Assay Kit (TaKaRa Bio, Beijing, China; \#T9300A) following the manufacturer's instructions. DNA and RNA in the LrEVs were quantified by using the Quant-iT ${ }^{\text {mix }}$ dsDNA Assay Kit (Invitrogen, \#Q33130) and Quant-iT ${ }^{\mathrm{m}}$ RNA Assay Kit (Invitrogen, \#Q33140) following the manufacturer's instructions, respectively. The contents of protein, DNA and RNA were normalized by using $1 \times 10^{11}$ particles of vesicles. The amount of LrEVs used in the following experiments was based on the protein content.

\section{Proteomic analysis}

Triplicate biological LrEVs samples were sent to Hangzhou PTM Biolabs (Hangzhou, Zhejiang Province, China) for proteomic analysis. In brief, the LrEVs samples were lysed by sonication on ice in lysis buffer $(8$ $\mathrm{mol} / \mathrm{L}$ urea, $1 \%$ protease inhibitor cocktail, $2 \mathrm{mmol} / \mathrm{L}$ EDTA), and the protein supernatants were harvested by centrifugation $\left(12,000 \times g, 15 \mathrm{~min}, 4^{\circ} \mathrm{C}\right)$. The extracted protein solutions were digested with trypsin (Promega, \#V5111) at a 1:50 (w/w; trypsin to protein) overnight at $37^{\circ} \mathrm{C}$. After fractionating by the high $\mathrm{pH}$ reverse-phase HPLC, the tryptic peptides were processed by tandem mass spectrometry (MS/MS) in Q ExactiveTM Plus (Thermo) coupled online to the UPLC (LC-MS/MS). The resulting spectrum was analyzed against $L$. reuteri genome draft sequence in the UniProt database. The subcellular localization of the identified proteins was predicted by the CELLO v.2.5 online tool (set for Grampositive bacteria; http://cello.life.nctu.edu.tw/). The biological function of the identified proteins was classified by Gene Ontology (GO) annotation from the UniProtGOA database (http://www.ebi.ac.uk/GOA/). If some identified proteins were not annotated by UniProt-GOA database, the InterProScan soft (http://www.ebi.ac.uk/ interpro/) would be used to annotate protein's GO function based on the protein sequence alignment method. All parameter settings and bioinformatic annotations were performed as described in our previous study [27].

\section{Animals and housing}

Newly hatched broiler chicks (Arbor Acres) were obtained from Dacheng Poultry Industry Company (Xianyang, Shaanxi Province, China). The chicks were housed in stainless-steel cages in a sterilized room with filtered air, strict sanitary conditions and age-appropriate temperatures. All chicks were fed an age-appropriate commercial diet containing no antibiotic additives. Drinking water and diets were offered ad libitum. All procedures of animal experiments were approved by the Ethics Committee of Animal Care and Use at Northwest A\&F University. 


\section{LPS-induced intestinal inflammation in broiler chicken model}

A total of 144 broiler chicks (1 male: 1 female) were randomly selected and grouped by body weight into 4 treatment groups when they were 7 days of age including: (1) a negative control challenged with PBS (group PBS; average $\mathrm{BW}=136.3 \pm 3.5 \mathrm{~g}$ ); (2) a positive control challenged with LPS (group LPS; average BW = 137.1 \pm 3.1 g); (3) a group treated with $L$. reuteri $\mathrm{BBC} 3$ and challenged with LPS (group LR + LPS; average BW $=136.8 \pm 2.8 \mathrm{~g}$ ); (4) a group treated with LrEVs and challenged with LPS (group LrEVs+LPS; average BW $=135.9 \pm 3.4 \mathrm{~g}$ ). All birds were placed in two-level wired cages and each group had 6 cages (replicates; 3 male and 3 female) of 6 birds each. Three replicates per treatment group were distributed in the upper cages and the other three replicates per treatment group were distributed in the lower cages. At 12, 14 and 18 days of age, the birds in the three LPS-challenged groups were intraperitoneally injected LPS $(500 \mu \mathrm{g} / \mathrm{bird}$; E. coli O111:B4 origin; Sigma, \#L4391) in $100 \mu \mathrm{L}$ PBS; birds in the PBS group were intraperitoneally injected an equal volume of sterile PBS. The dosage of LPS challenge was determined based on previous reports [29]. Every other day from 7 to 21 days of age, the birds in the LR + LPS and LrEVs+LPS groups were given by gavage the cultured $L$. reuteri BBC3 $\left(5 \times 10^{9} \mathrm{CFU} /\right.$ bird $)$ and purified LrEVs $(200 \mu \mathrm{g} /$ bird) in $200 \mu \mathrm{L}$ protectant (5\% skim milk), respectively; the birds in the remaining two groups were given by gavage an equal volume of PBS in protectant. The chicks were sacrificed by jugular exsanguination after intravenous injection of pentobarbital sodium $(20 \mathrm{mg} / \mathrm{kg}$ body weight) on day 21 for sample collection.

\section{Growth performance and intestinal morphology analysis}

The body weight, feed intake and the number of deaths were recorded on a replicate basis and used to calculate the growth performance of birds from 7 to 21 days of age, including average daily weight gain (ADWG), average daily feed intake (ADFI), feed gain ratio (F/G). ADWG, ADFI and F/G were adjusted when any bird died. Jejunum tissues were sampled at 21 days of age and fixed in neutral-buffered $4 \%$ paraformaldehyde solution. The fixed tissues were dehydrated through a graded series of ethanol (50\%, 70\%, 85\%, 95\% and 100\% alcohol), embedded into paraffin and sectioned at a thickness of $3-5 \mu \mathrm{m}$. After staining with hematoxylin and eosin, the sections were examined with an Olympus BX53F microscope (Olympus, Tokyo, Japan) at 20x magnification. Villus height and crypt depth were measured from ten representative well-preserved villi per segment, then the ratio of villus height to crypt depth (VH/CD) was calculated.

\section{In vitro macrophage assay}

HD11 cells, a transformed chicken M $\phi$ cell line derived from bone marrow [30], were used in this study and cultured in the complete PRMI-1640 medium (Gibco, \#22400089) supplemented with 10\% heat-inactivated fetal bovine serum (FBS; Zeta-Life, \#Z7181FBS-500), $100 \mathrm{U} / \mathrm{mL}$ penicillin and $100 \mu \mathrm{g} / \mathrm{mL}$ streptomycin (Sigma, \#P4333) in an atmosphere of $5 \% \mathrm{CO}^{2}$ at $37^{\circ} \mathrm{C}$. Visualization of the internalization of LrEVs by HD11 cells was performed as presented in our previous study [27]. Briefly, HD11 cells $\left(5 \times 10^{5}\right.$ cells $\left./ \mathrm{mL}\right)$ were coincubated with DiI (Sigma, \#42364)-labeled LrEVs $(10 \mu \mathrm{g} / \mathrm{mL})$ for $6 \mathrm{~h}$. After washing and staining with $4^{\prime}$, move to the same line (DAPI; Sigma, \#D9542), the cells were detected using the high-speed spinningdisk confocal microscope (Andor Revolution XD, Andor Technology, UK).

For the LPS-challenged M $\phi$ assay, HD11 cells $\left(5 \times 10^{5}\right.$ cells $/ \mathrm{mL})$, were pretreated with PBS or LrEVs $(10 \mu \mathrm{g} /$ $\mathrm{mL})$ for $12 \mathrm{~h}$ and stimulated with PBS or LPS $(1 \mu \mathrm{g} / \mathrm{mL})$ for $12 \mathrm{~h}$. The cells were collected for the determination of cytokine gene expression, NF- $\mathrm{kB}$ p65 transcription factor activity and cell viability. Three independent experiments were performed per treatment.

\section{In vitro $\mathbf{M} \varphi$-splenic lymphocyte coculture}

Splenic lymphocytes were isolated from the spleen of chickens in the LPS group at day 21 using the Chicken Splenic Lymphocyte Isolation Kit (Solarbio, \#P9120) according to the manufacturer's instructions. The isolated splenic lymphocytes were maintained in complete PRMI-1640 medium supplemented with 10\% FBS, 100 $\mathrm{U} / \mathrm{mL}$ penicillin and $100 \mu \mathrm{g} / \mathrm{mL}$ streptomycin in an atmosphere of $5 \% \mathrm{CO}^{2}$ at $37^{\circ} \mathrm{C}$. For transwell culture, LrEVs- or PBS-pretreated HD11 cells $(10 \mu \mathrm{g} / \mathrm{mL}$ LrEVs, $5 \times 10^{5}$ cells $/ \mathrm{mL}, 12 \mathrm{~h}$ ) were added to the basolateral compartment of a 6-well plate, and splenic lymphocytes $\left(5 \times 10^{5}\right.$ cells $\left./ \mathrm{mL}\right)$ were seeded on the apical compartment of 6-well Hanging Inserts $(0.4 \mu \mathrm{m}$; Corning, \#M150865). After 12-h coculture, splenic lymphocytes were collected to determine the cytokine gene expression. Three independent experiments were performed per treatment.

\section{Enzymatic treatment of LrEVs}

To remove vesicular nucleic acids and proteins, $1 \mathrm{mg} /$ $\mathrm{mL}$ of native LrEVs were treated with $10 \mathrm{U} / \mathrm{mL}$ of DNase I (Thermo Scientific, \#EN0521), $10 \mathrm{U} / \mathrm{mL}$ of RNase I (Solarbio, \#R8021), and $1 \mathrm{mg} / \mathrm{mL}$ of proteinase K-agarose (Sigma, \#P9290), respectively, as described previously $[16,31]$. The native LrEVs were incubated with DNase I and RNase I at $37^{\circ} \mathrm{C}$ for $30 \mathrm{~min}$, and with proteinase $\mathrm{K}$-agarose at $37^{\circ} \mathrm{C}$ for $2 \mathrm{~h}$. The enzymes were inactivated at $75^{\circ} \mathrm{C}$ for $1 \mathrm{~h}$. Proteinase $\mathrm{K}$-agarose was 
additionally removed by centrifugation $(12,000 \times g, 1$ $\min )$.

\section{Ex vivo jejunum explant culture}

The culture of jejunum tissues was performed as described previously [32]. Briefly, jejunum explants (5 $\mathrm{mm} \times 5 \mathrm{~mm}$ ) without lesions from healthy broilers of 21 days old were rinsed under sterile saline solution at $4{ }^{\circ} \mathrm{C}$ and maintained in 24-well culture plates with the complete PRMI-1640 medium supplemented with $10 \%$ FBS, $0.25 \mu \mathrm{g} / \mathrm{mL}$ fungizone (Sigma-Aldrich, \#V900919), $100 \mathrm{U} / \mathrm{mL}$ penicillin and $100 \mu \mathrm{g} / \mathrm{mL}$ streptomycin (in an atmosphere of $5 \% \mathrm{CO}^{2}$ at $37^{\circ} \mathrm{C}$ ). The explants were pretreated with native LrEVs $(10 \mu \mathrm{g} / \mathrm{mL})$, DNase I and RNase I-treated LrEVs (DR-LrEVs; $10 \mu \mathrm{g} / \mathrm{mL}$ ) or proteinase K-agarose-treated LrEVs (PK-LrEVs; $10 \mu \mathrm{g} / \mathrm{mL}$ before proteinase K-agarose treatment) for $6 \mathrm{~h}$ and then exposed to LPS $(1 \mu \mathrm{g} / \mathrm{mL})$ for $6 \mathrm{~h}$. The explants were collected to determine cytokine gene expression and myeloperoxidase (MPO) activity. Three independent experiments were performed per treatment.

\section{Quantitative real-time PCR (qRT-PCR)}

Total RNA was extracted from jejunum tissues, HD11 cells, splenic lymphocytes and jejunum explants using the Total RNA Kit (Omega Bio-Tek, \#R1034) following the manufacturer's instructions. After examination of RNA purity and quality, qRT-PCR analysis was conducted with One Step SYBR ${ }^{\circ}$ PrimeScript ${ }^{\mathrm{m}}$ PLUS RTPCR Kit (TaKaRa Bio, Beijing, China; \#RR096A) in a Real-Time PCR Detection System (CFX96 Touch, BioRad) according to the manufacturer's instructions. The primer sequences of target genes and a reference gene $(\beta$-actin) used for qPCR are shown in Supplementary Table S1. Triplicate qRT-PCR reactions for each sample were conducted under the following settings: $95^{\circ} \mathrm{C}$ for 1 min, 40 cycles of $95^{\circ} \mathrm{C}$ for $15 \mathrm{~s}$ and $60^{\circ} \mathrm{C}$ for $30 \mathrm{~s}$. Relative mRNA expression was calculated by the method of the $2^{-\Delta \Delta \mathrm{Ct}}$ as described previously [33], and expressed as the fold-change relative to the control, which was normalized to 1 .

\section{NF-KB p65 activity assay}

The NF- $\mathrm{kB}$ p 65 transcription factor activity in the cell nucleus from chicken $M \phi$ was measured using the NFкB p65 Transcription Factor Assay Kit (Abcam, \#ab133112) following the manufacturer's instructions with some modifications. Briefly, nuclear protein extracts were prepared from cultured $\mathrm{M} \phi$ using the Nuclear Extraction Kit (Abcam, \#ab113474) according to the manufacturer's instructions. The extracted nuclear fractions were added into black 96-well plates containing the consensus binding sequence for NF- $\mathrm{KB}$ p65 and incubated for $1 \mathrm{~h}$ at room temperature. After washing, a chicken reactive rabbit anti-NF-kB p65 antibody (Abcam, \#ab16502) was loaded into each well and incubated for $1 \mathrm{~h}$ at room temperature. After washing, the HRPconjugated secondary goat anti-rabbit antibody was added into each well and incubated for $1 \mathrm{~h}$ at room temperature. Each well was washed 5 times and loaded with a developing solution and then incubated for 15$45 \mathrm{~min}$ at room temperature. The $\mathrm{OD}_{450}$ values were measured using a Microplate Reader (Epoch 2, Biotek) following the addition of stop solution to each well. Each sample was determined in triplicate.

\section{Cell viability}

The viabilities of HD11 cells were determined by Trypan Blue dye (Sigma, \#T6146) exclusion assay as described previously [34]. Data were presented as the proportion (\%) of viable cells (cells excluding blue dye) to the total counted cells.

\section{MPO activity assay}

Jejunum tissues or explants were collected to determine the MPO activity using the Myeloperoxidase Assay Kit (Nanjing Jiancheng Bioengineering Institute, Jiangsu, China; \#A044-1-1) following the manufacturer's instructions. Data were presented as a unit of MPO activity (U) per $\mathrm{mg}$ of protein. One $\mathrm{U}$ is defined as the amount of enzyme that catalyzes $1 \mu \mathrm{mol}$ peroxide per minute at $37^{\circ} \mathrm{C}$.

\section{Statistical analysis}

All data were expressed as mean \pm standard error of the mean (SEM). Student's $t$-test was used for the analysis of differences between the two groups. One-way ANOVA with Newman-Keuls test as the post hoc test was performed for comparisons among greater than two groups. Statistical significance was declared at $P<0.05$. All analyses were performed using Graph Pad Prism software 5.0 (San Diego, CA, USA).

\section{Results}

\section{L. reuteri $\mathrm{BBC} 3$ releases nanosized extracellular vesicles}

The 16S rRNA sequence-based phylogenetic analysis indicated that the Lactobacillus isolate used in this study belonged to a subclade of $L$. reuteri, showing $99.2-99.5 \%$ similarity to other strains within $L$. reuteri (Fig. 1a). Ultrastructural analysis of the isolate, L. reuteri $\mathrm{BBC}$, showed that single vesicles, occasionally multiple vesicles, shed from the surface of individual bacterial cells (Fig. 1b). As shown in Fig. 3c, these vesicles were obtained from the culture supernatants of $L$. reuteri $\mathrm{BBC} 3$ using a series of filtration and centrifugation steps. A large number of vesicle particles were found in fractions 3-5 following purification by density gradient centrifugation (Fig. 1d). The density range for these fractions 


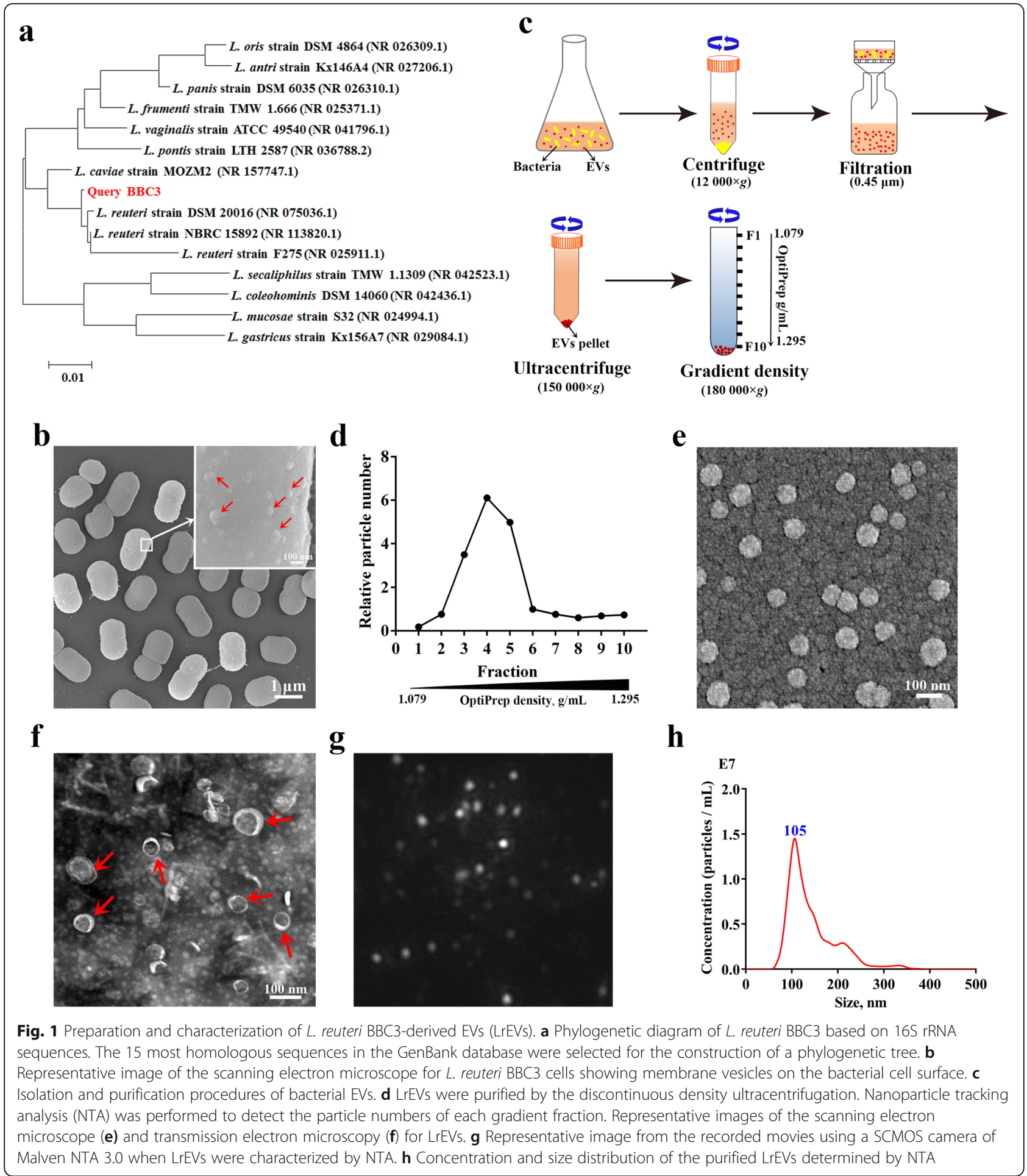

was from 1.127 to $1.199 \mathrm{~g} / \mathrm{mL}$. These EV-containing fractions were pooled and subsequently visualized by scanning electron microscopy (Fig. 1e) and transmission electron microscopy (Fig. 1f). These micrographs revealed that the purified LrEVs were membrane-enclosed structures with spherical morphology, and the majority of these vesicles ranged from $50 \mathrm{~nm}$ to $150 \mathrm{~nm}$ in diameter. These findings are consistent with the typical results characterized by NTA analysis (Fig. $1 \mathrm{~g}$ and f), which showed that the sizes of LrEVs ranged from 60 to 
$250 \mathrm{~nm}$ and peaked at $105 \mathrm{~nm}$. Together, these results demonstrate that this $L$. reuteri $\mathrm{BBC} 3$ isolate can release nano-sized vesicles.

\section{Proteomic analysis of LrEVs}

Biochemical analysis revealed that the LrEVs contained DNA, RNA and proteins, and the protein content was noticeably higher than that of DNA or RNA (Fig. 2a). These findings are in accordance with the previously described composition of EVs derived from other Lactobacillus strains [12, 14]. Proteomic analysis was further performed to detect the protein profile of LrEVs. A total of 92 overlapping proteins were identified in triplicate biological LrEVs samples and selected for further analysis (Fig. 2b). The subcellular localization of these identified proteins showed that $56.5 \%$ was originated from the cytoplasm and $43.5 \%$

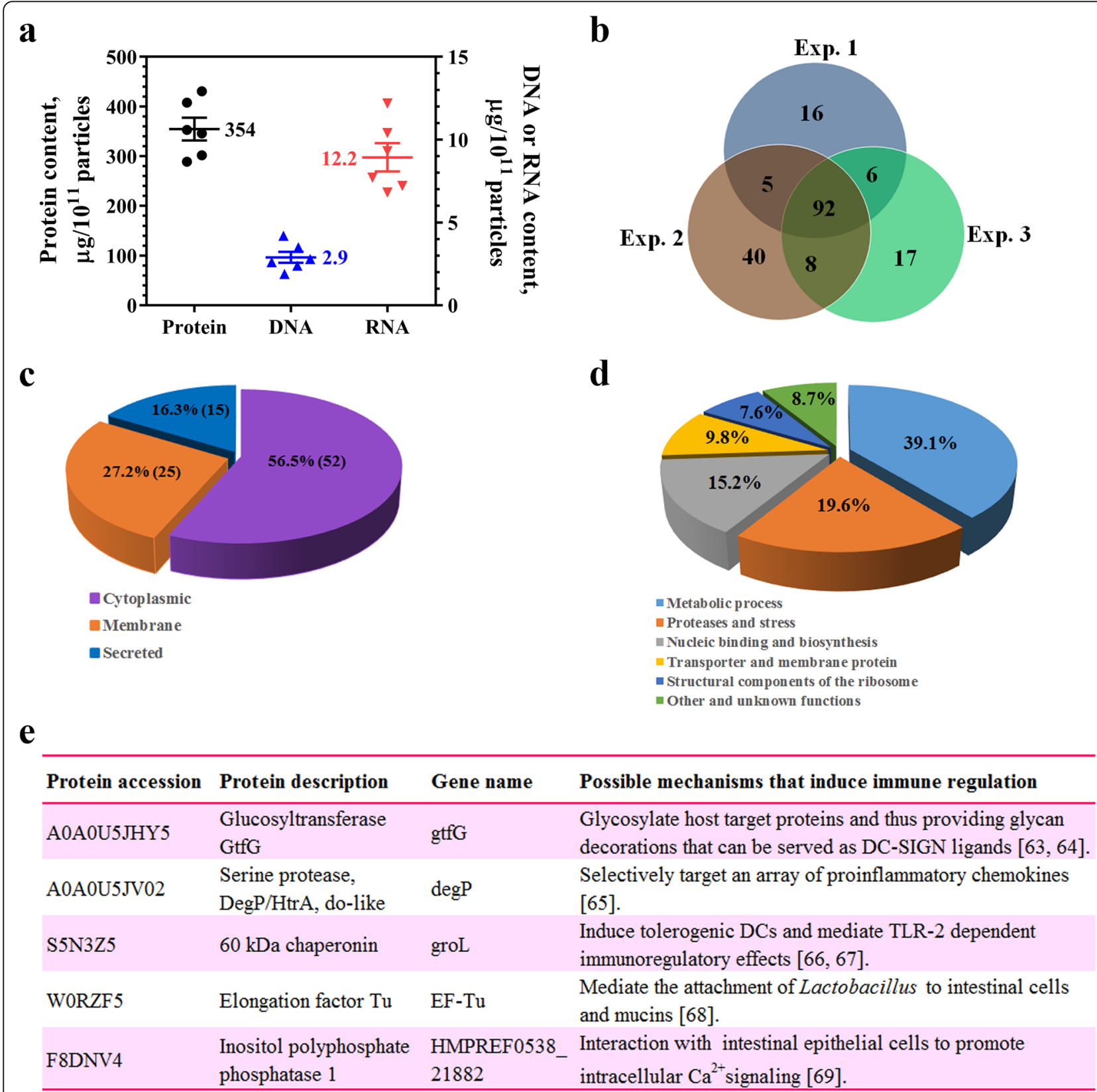

Fig. 2 Biochemical and proteomic analyses of LrEVs. a Quantifications of protein, DNA and RNA in the LrEVs. b Venn diagram showed that 92 overlapping proteins were identified in triplicate samples. c Subcellular localization of the identified proteins present in the LrEVs. It was found that $56.5 \%$ was cytoplasmic and $43.5 \%$ belonged to the membrane and secreted proteins. $\mathbf{d}$ Biological function classification of the identified proteins. It was found that the majority was either metabolic process (39.1\%) or proteases and stress (19.6\%). e A selected list of the identified proteins which may function in immune regulation, including protein accession, protein description and possible mechanisms 
belonged to membrane proteins and secreted proteins (Fig. 2c). According to the distribution of biological functions analyzed by GO annotation, most of these proteins were classified into metabolic enzymes, proteases, nucleicbinding proteins, transporter and membrane proteins and structural components of the ribosome, suggesting that LrEVs may be involved in metabolism, transporter activity, translation and transcription, signaling and stress, etc. (Fig. $2 \mathrm{~d})$. The detailed information of all identified proteins is listed in Supplementary Table S2. Notably, several homologous proteins that were previously described as mediators of anti-inflammatory or beneficial effects in other probiotics or commensal bacterium were also observed in LrEVs, such as glucosyltransferase, serine protease, $60 \mathrm{kDa}$ chaperonin, elongation factor $\mathrm{Tu}$ and inositol polyphosphate phosphatase 1 (Fig. 2e). Altogether, these findings reveal that LrEVs carry DNA, RNA and some immunoregulatory proteins, leading us to investigate the functions of these vesicles in the underlying mechanism of bacterium-host interactions.

\section{L. reuteri $\mathrm{BBC} 3$ and its EVs attenuate LPS-induced intestinal injury in chickens}

To investigate whether $L$. reuteri $\mathrm{BBC} 3$ and $\mathrm{LrEV}$ s have therapeutic effects, broiler chickens were given $L$. reuteri BBC3 or LrEVs by gavage during LPS-induced inflammation (Fig. 3a). Three days post the last LPS injection, ADWG $(P<0.001)$ and ADFI $(P<0.01)$ of LPS-only group (Fig. 3b; PBS + LPS) were significantly lower than those of PBS-only group (Fig. 3b; PBS + PBS); while F/G $(P<0.001)$ and the cumulative mortality $(P<0.01)$ of LPS-only group were significantly higher than those of PBS-only group. Remarkably, administration of both $L$. reuteri $\mathrm{BBC} 3$ and LrEVs significantly attenuated the reduced ADWG $(P<0.001)$ and ADFI $(P<0.01)$ and the increased F/G $(P<0.001)$ and mortality $(P<0.05)$ caused by LPS challenge (Fig. 3b; LR + LPS and LrEVs+LPS). According to histological analysis of jejunum tissues, obvious symptoms of intestinal injury, such as thinner mucosa, deformed crypt and many swelled and shed villus, were found in the LPS-only group; whereas, administration of $L$. reuteri $\mathrm{BBC} 3$ and LrEVs reduced the occurrence of intestinal injury (Fig. 3c). Additionally, LPS challenge significantly decreased the villus height $(P<0.001)$ and the value of $\mathrm{VH} / \mathrm{CD}(P<0.001)$, and significantly increased the crypt depth $(P<0.001)$; while administration of L. reuteri $\mathrm{BBC} 3$ and LrEVs significantly ameliorated the reduced villus height $(P<0.001)$ and $\mathrm{VH} / \mathrm{CD}(P<0.01)$ and the increased crypt depth $(P<0.01)$ (Fig. $4 \mathrm{~d})$. Interestingly, growth performance, mortality and indicators of villus morphology in the LrEVs+LPS group were not significantly different $(P>0.05)$ from those in the LR + PBS group, indicating that LrEVs had similar effects as $L$. reuteri $\mathrm{BBC} 3$ in attenuating LPSinduced intestinal injury.
LrEVs modulate the gene expression of pro- and antiinflammatory mediators in jejunum tissues

As shown in Fig. 4a, compared with the PBS-only group, the gene expression of several pro-inflammatory mediators, including TNF- $\alpha(P<0.001), I L-1 \beta(P<0.001), I L-6$ $(P<0.001), I L-8(P<0.001), I L-17(P<0.001)$ and MIP$1 \beta(P<0.001)$, were significantly elicited in the LPS-only group. However, compared with the LPS-only group, administration of LrEVs significantly inhibited the gene expression of TNF- $\alpha(P<0.001), I L-1 \beta(P<0.001), I L-6$ $(P<0.05), I L-8(P<0.01), I L-17(P<0.001)$ and $M I P-1 \beta$ $(P<0.01)$, implying that LrEVs might mediate protection in LPS-challenged chickens through suppressing these pro-inflammatory mediators. Besides, the gene expression of $I L-10(P<0.01)$ and TGF- $\beta(P<0.01)$ in the LrEVs+LPS group were significantly higher than those in the LPS-only group, indicating that these antiinflammatory cytokines were also involved in the LrEVsmediated regulation of intestinal inflammation. Meanwhile, LrEVs induced stronger suppression of TNF- $\alpha$ gene expression $(P<0.05)$ and enhancement of $I L-10$ gene expression $(P<0.01)$ than LR. Furthermore, the MPO activity, representing neutrophil infiltration into inflamed tissues [35], was also significantly inhibited by L. reuteri BBC3 $(P<0.001)$ and LrEVs $(P<0.001)$. Accordingly, we conclude that the regulation of intestinal inflammation by $L$. reuteri $\mathrm{BBC} 3$ and LrEVs correlated with the suppression of pro-inflammatory cytokines and the activation of anti-inflammatory cytokines.

\section{LrEVs suppress NF-KB-dependent pro-inflammatory responses in LPS-activated chicken macrophages}

The similar protective effects mediated by $L$. reuteri $\mathrm{BBC} 3$ and LrEVs suggest that L. reuteri $\mathrm{BBC} 3$ might function via LrEVs on the host. To further investigate the roles of LrEVs in the microbiota-host interactions, we first examined whether LrEVs could be taken up by chicken HD11 M $\phi$ in vitro. After co-incubation with HD11 cells for $6 \mathrm{~h}$, the DiI-labeled LrEVs (red signals) were found in the cytoplasm of these cells, suggesting that LrEVs were internalized by $\mathrm{M} \phi$ (Fig. 5a). Moreover, pretreatment with LrEVs markedly inhibited the elevated activity of NF-KB $(P<0.001)$ in LPS-activated HD11 cells (Fig. 5b). Meanwhile, compared with stimulation with only PBS in HD11 cells, stimulation with only LPS significantly up-regulated the gene expression of NF-kBdependent pro-inflammatory cytokines TNF- $\alpha$ $(P<0.001), \quad I L-1 \beta \quad(P<0.001)$ and $I L-6 \quad(P<0.001)$; stimulation with only LrEVs significantly enhanced the gene expression of anti-inflammatory cytokines $I L-10$ $(P<0.01)$ and TGF- $\beta(P<0.001)$. In LPS-challenged HD11 cells, pretreatment with LrEVs significantly induced lower gene expression of TNF- $\alpha(P<0.001), I L-1 \beta$ $(P<0.001)$ and IL-6 $(P<0.001)$ but higher gene 


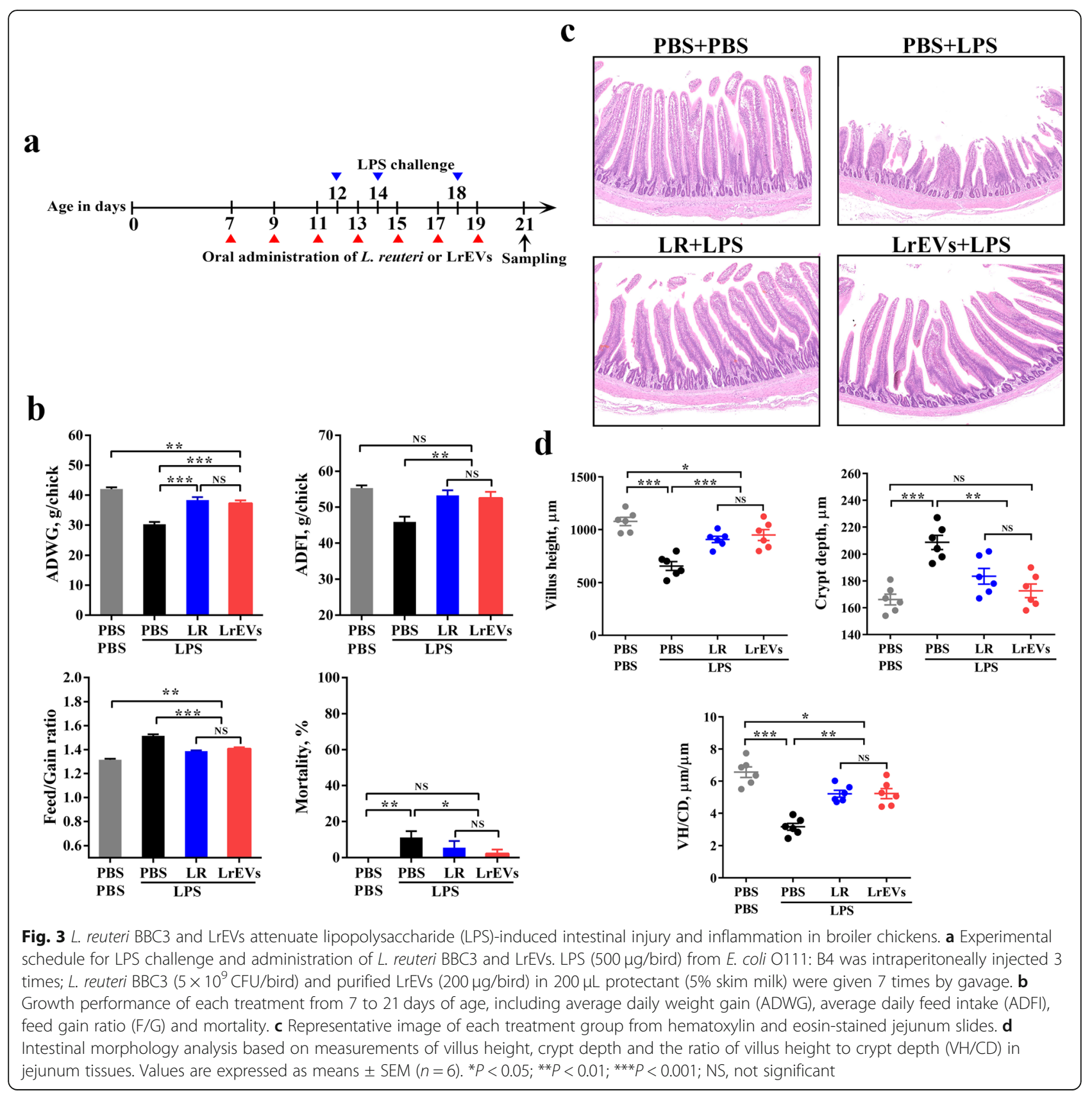

expression of $I L-10(P<0.001)$ and TGF- $\beta(P<0.001)$ than pretreatment with PBS (Fig. 5c). Additionally, LrEVs did not affect $(P>0.05)$ the viability of PBS- or LPS-treated cells, thus ruling out the possibility that cytotoxicity was responsible for LrEVs-mediated suppression of NF- $\mathrm{KB}$ activity and pro-inflammatory cytokines. LPS stimulation, but not LrEVs-derived factors, was associated with the reduced cell viability (Fig. 5d). Collectively, these data demonstrate the capacity of internalized LrEVs in the suppression of NF-kB-dependent pro-inflammatory responses in LPS-activated $\mathrm{M} \phi$.
LrEVs induce anti-inflammatory responses in splenic lymphocytes through the activation of macrophages To investigate the crosstalk between LrEVs, antigenpresenting cells (APCs) and adaptive immune cells, we performed an in vitro coculture assay of splenic lymphocytes with LrEVs-pretreated $M \phi$ using a Transwell system (Fig. 6a). The HD11 cells were pretreated with LrEVs for $12 \mathrm{~h}$, washed and subsequently cocultured with splenic lymphocytes of LPS-challenged chickens for $12 \mathrm{~h}$. Splenic lymphocytes cocultured with LrEVspretreated $\mathrm{M} \phi$ showed the enhanced gene expression of IL-10 $(P<0.001)$ and TGF- $\beta(P<0.001)$ compared to 


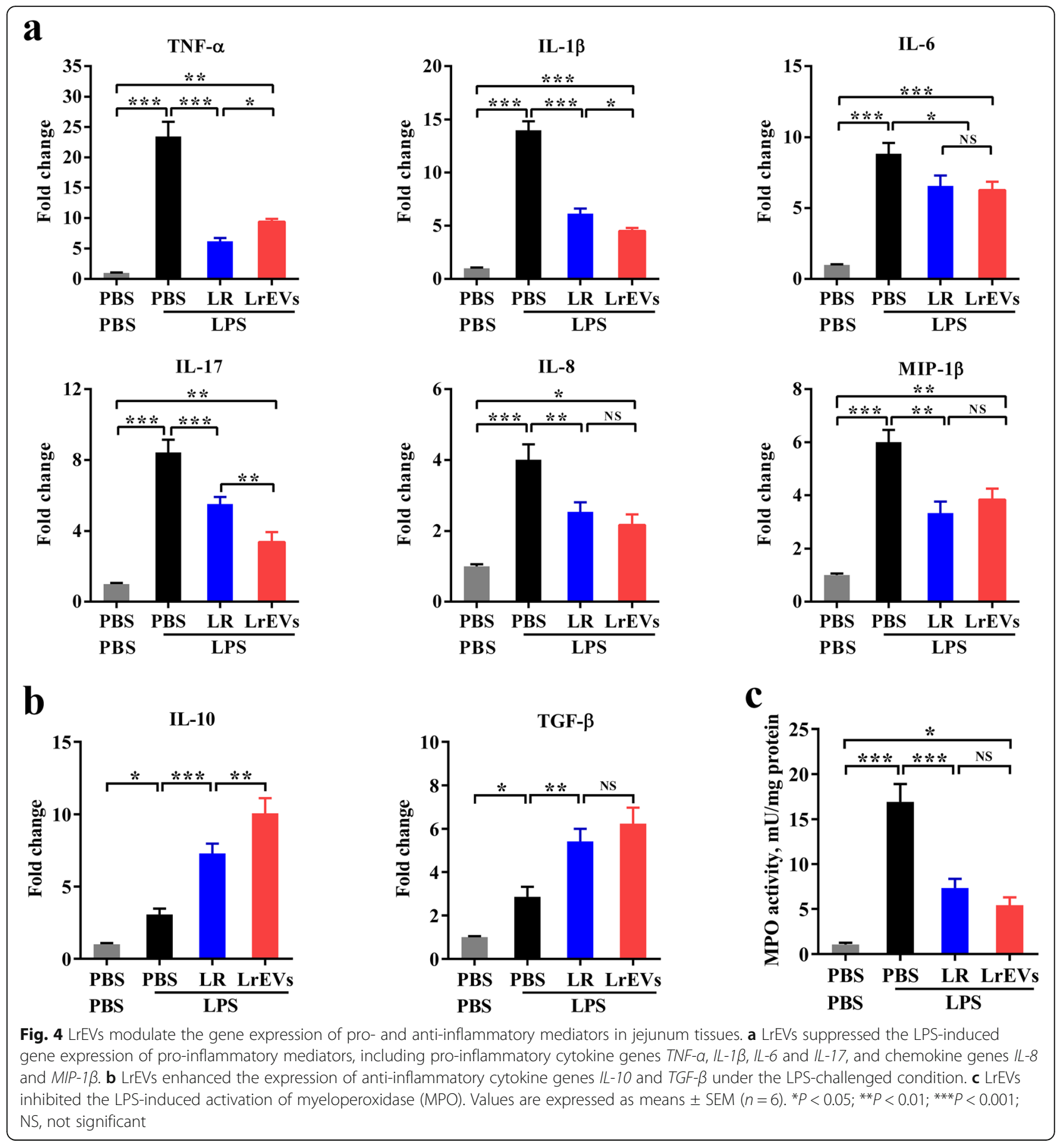

these cells cocultured with PBS-pretreated M $\phi$ (Fig. 6b). Moreover, LrEVs significantly downregulated the gene expression of IFN- $\gamma(P<0.001)$ and $I L-17(P<0.001)$, the representative Th1 and Th17 cytokines, in splenic lymphocytes cocultured with $\mathrm{M} \phi$; while the gene expression of IL-4 $(P>0.05)$, a representative Th2 cytokine, was not affected by LrEVs (Fig. 6c). Furthermore, LrEVs could also enhance the gene expression of $C D 25(P<0.001)$, T-lymphocyte antigen 4
(CTLA-4, $P<0.001)$ and lymphocyte activation gene 3 (LAG-3, $P<0.001)$ in splenic lymphocytes cocultured with $\mathrm{M} \phi$ (Fig. 6d).

Both vesicular proteins and nucleic acids are essential for the LrEVs-mediated immunoregulation

We further used an ex vivo jejunum explant culture model to investigate the potential roles of these vesicular molecules in LrEVs-mediated regulation. LrEVs were 


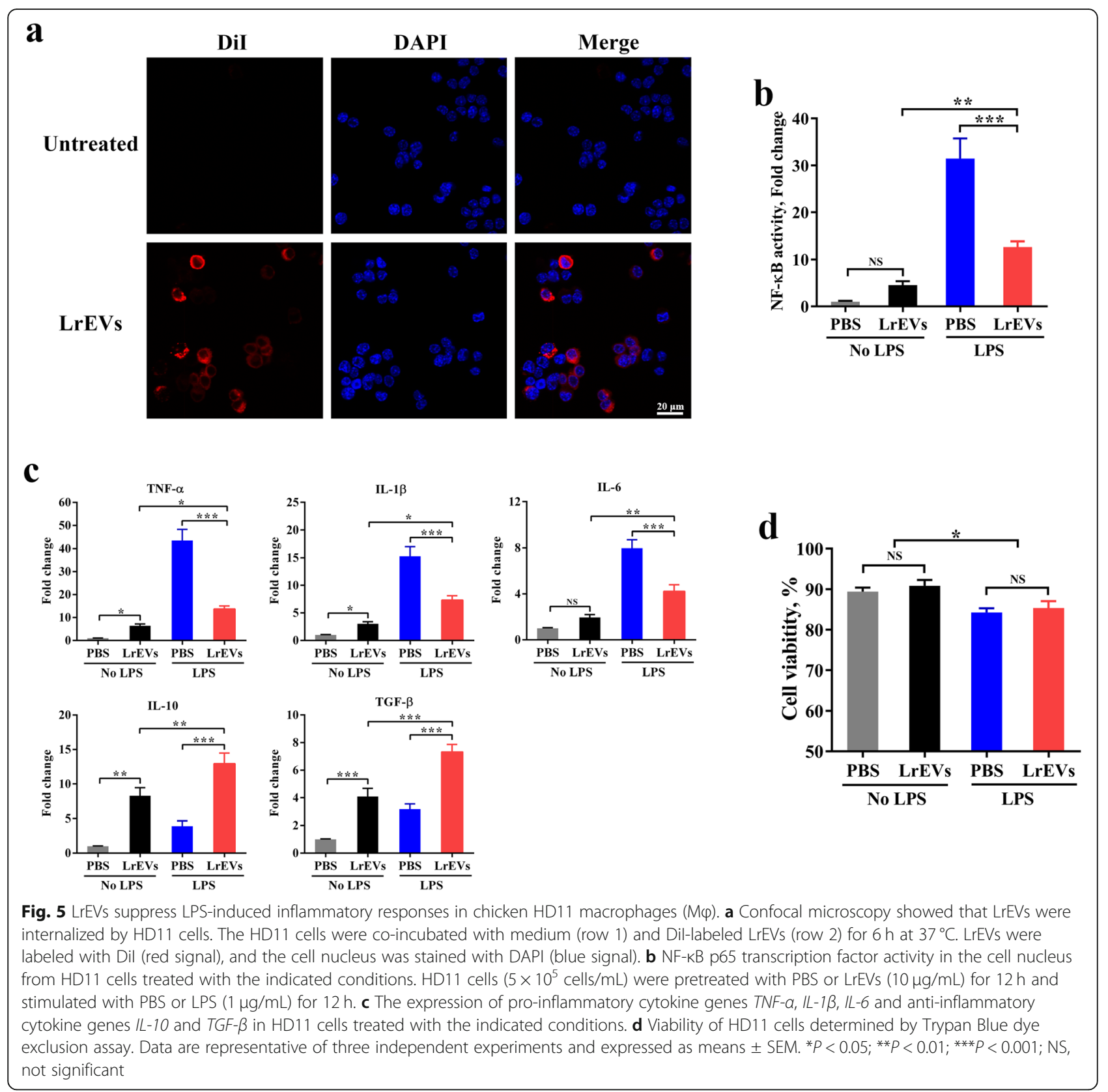

treated with DNase I and RNase I (DR) and proteinase $\mathrm{K}$-agarose (PK) to remove nucleic acids and proteins, respectively. The enzymes were inactivated at $75^{\circ} \mathrm{C}$ for $1 \mathrm{~h}$ and proteinase $\mathrm{K}$-agarose was additionally removed by centrifugation. Surprisingly, although these treatments significantly decreased the content of vesicular DNA $(P<0.05)$, RNA $(P<0.05)$ or proteins $(P<0.001)$ to some extent, they did not remove these components as much as expected, implying that the signals delivered by EVs are highly protected against exogenous proteases (Fig. 7a). Besides, the reduced content of nucleic acids or proteins might affect the functions of native LrEVs. Subsequently, a jejunum tissue culture system with LPS challenge was used as a closer model to the LPSchallenged condition in vivo. During ex vivo jejunum explant culture with LPS challenge, DR treatment significantly decreased the suppression of native LrEVs on the gene expression of TNF- $\alpha(P<0.01), I L-6(P<0.05)$ and IL-17 $(P<0.05)$, and PK treatment significantly decreased the suppression of native LrEVs on the gene expression of TNF- $\alpha(P<0.01), I L-6(P<0.01), I F N-\gamma(P<$ $0.05)$ and $I L-17(P<0.05)$ (Fig. 7b). Additionally, DRand PK-treated LrEVs showed a lower ability to induce the gene expression of $I L-10(P<0.001$ and $P<0.001)$ and TGF- $\beta(P<0.05$ and $P<0.001)$ compared to native LrEVs (Fig. 7c). Finally, DR and PK treatments also 


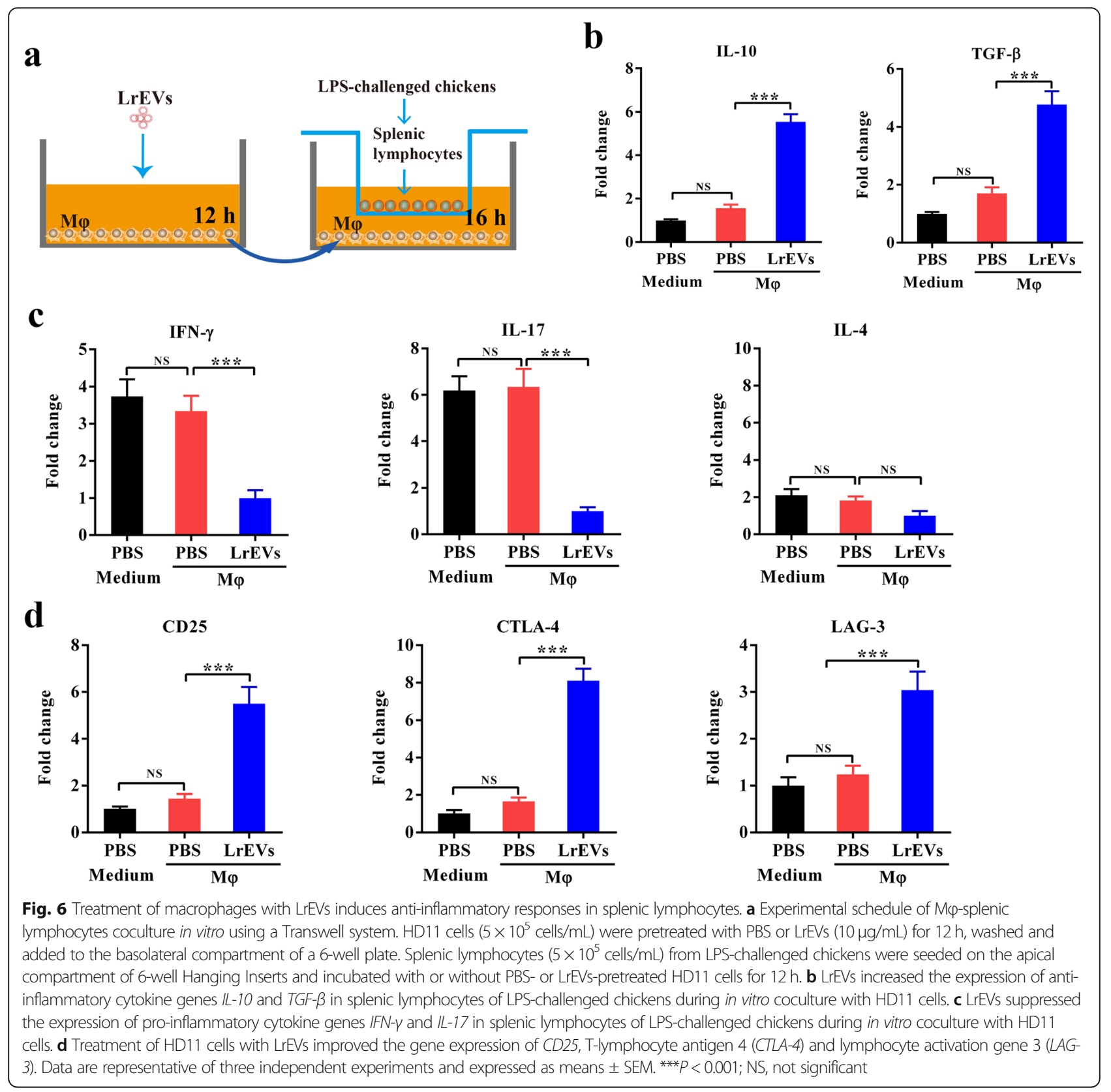

markedly reduced the ability of native LrEVs in the inhibition of MPO activity $(P<0.05$ and $P<0.001)$, indicating that these treatments significantly decreased the suppressive activity of native LrEVs to inflammatory responses. Collectively, we conclude that both vesicular proteins and nucleic acids are crucial in the LrEVsmediated immunoregulation.

\section{Discussion}

Prohibition of antibiotic growth promoters has promoted the development of nutritional immunomodulators as a particularly attractive strategy to maintain gut health in today's intensive poultry industry. Probiotics supplementation is becoming one of the most promising ways for the prevention and treatment of various inflammatory bowel diseases, due to its safety and ability in modulating intestinal immune homeostasis [36]. Previously, it has been illustrated that $L$. reuteri has the ability to regulate the host's immune system in humans and animals $[17,18]$. Consistent with these previous results, the present study demonstrated that administration of $L$. reuteri $\mathrm{BBC} 3$ attenuated the LPS-induced intestinal injury and inflammation in broiler chickens. We further revealed that EVs secreted by L. reuteri $\mathrm{BBC} 3$, a nanosized vesicle enriched with biological molecules of parental bacteria, could mediate bacteria-host interactions. 


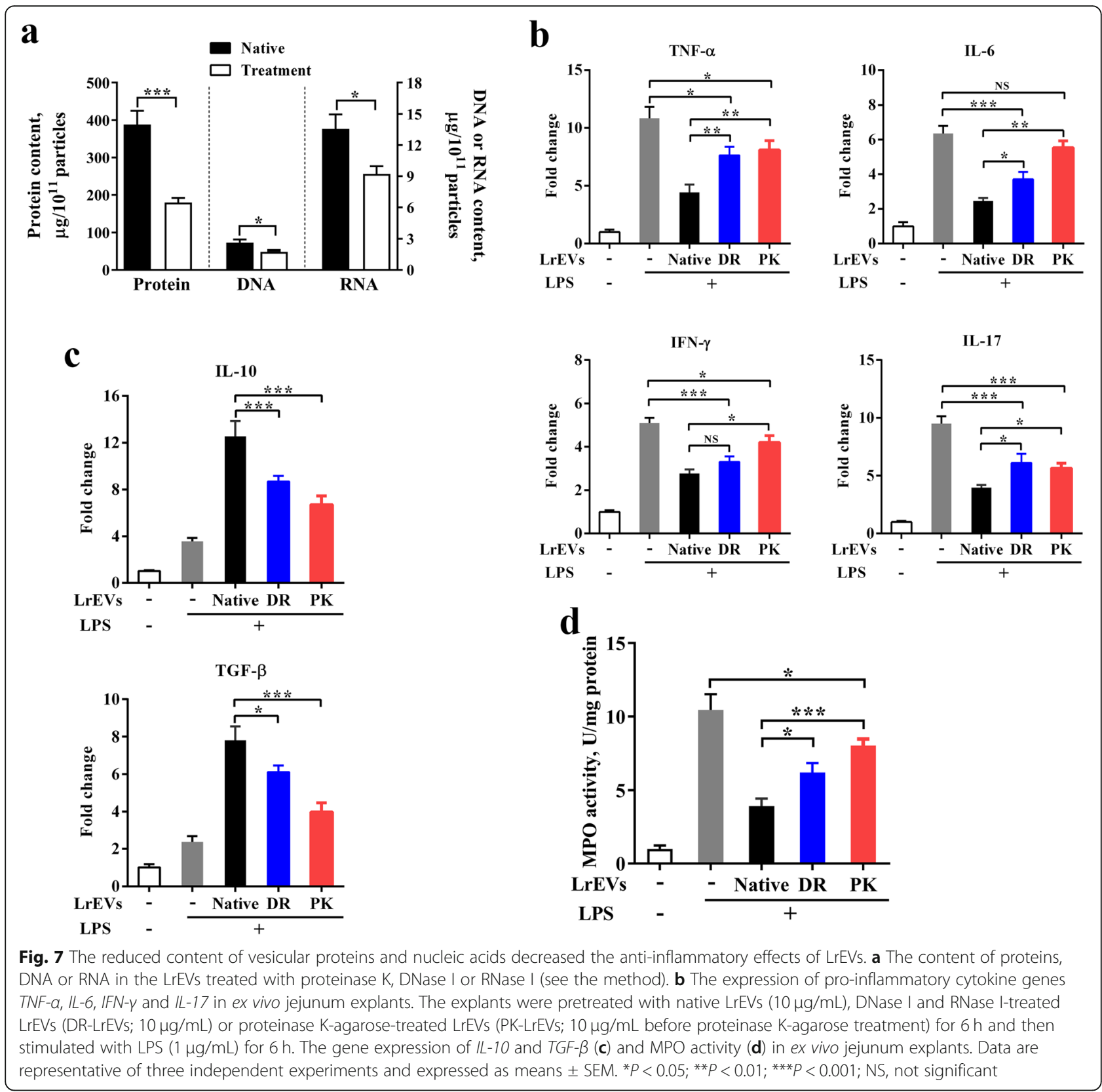

It is fundamentally important to understand the molecular mechanisms of the interaction of probiotics with the host to improve the application and clinical effectiveness of the probiotics. In addition to direct interaction with the host, the complex microbiota-host communication can also be mediated through active mediators secreted by microbiota, such as short-chain fatty acids, histamine and indole [37, 38]. Among various bacteriaderived mediators, EVs have been proved to play a key role in intercellular crosstalk or signaling [3, 39]. Bacterial EVs carry various bioactive molecules from parental bacteria, such as proteins, DNA, RNA and lipids. EVs are encapsulated vesicles with a nano-sized spherical lipid bilayer, thereby making them highly biocompatible and promoting the uptake by the host cells [4]. Much of the understanding of the role of bacterial EVs in mediating intercellular signaling is derived from numerous studies of pathogenic bacterial OMVs, showing that they are involved in the pathogenesis via delivering virulence factors to target cells $[40,41]$. Only recently has it been increasingly interested in the mechanism of EVs secretion by which probiotics and commensal microbiota communicate with the host. Previous studies have revealed that EVs derived from several probiotics and commensal bacteria, such as Bacteroides fragilis [42], Akkermansia muciniphila [43], Escherichia coli Nissle 
1917 [44], Bifidobacterium longum [45] and Lactobacillus paracasei [15], exerted similar effects as their parental bacteria. Consistent with these studies, we discovered that $L$. reuteri $\mathrm{BBC} 3$ could produce relatively high quantities of EVs, and treatment of broiler chicks with LrEVs reproduced the protective effects of $L$. reuteri $\mathrm{BBC} 3$ on LPS-induced lower performance and intestinal injury and inflammation.

Gram-negative bacterial LPS is known to induce acute inflammatory responses of the host by activating the NF- $\mathrm{kB}$ signaling pathway [46, 47]. The relatively high dose of LPS $(500 \mu \mathrm{g} / \mathrm{bird})$ was used to induce an in vivo model of intestinal inflammation in the present study. Previous studies have shown that the recovery of intestinal mucosal is relatively slow after a high dose of LPS challenge [48-50]. Consistent with these findings, our results showed that the high mortality and significant intestinal injury were observed in the LPS group continued until the third day after LPS injection. In the model of LPS-induced intestinal injury, many inflammatory mediators such as pro-inflammatory cytokines TNF- $\alpha$, IL- $1 \beta$ and IL-6 and chemokines IL-8, were induced and released in the intestinal tissue $[47,51]$. As a key mediator involved in inflammatory responses, IL-17 causes tissue damage via recruiting neutrophils into the gut and promoting the release of pro-inflammatory cytokines [42]. Macrophage inflammatory protein (MIP)- $1 \beta$ is particularly concerning chemokine associated with a variety of pro-inflammatory activities [52]. Our results showed that treatment of broiler chicks with LrEVs could attenuate LPS-induced intestinal inflammation by inhibiting the expression of these pro-inflammatory mediators and improving the expression of anti-inflammatory cytokines. Meanwhile, the different degrees of regulation of gene expression indicated that the induced immunomodulatory effectiveness between bacterial EVs and the whole bacteria are not exactly equal. Despite this fact, these available results allow us to believe that EVs may act as an important mechanism of interaction between microbiota and the host.

Accordingly, it is conceivable that LrEVs could modulate the functions of the host's immune cells. Indeed, we found that LrEVs could effectively be internalized by chicken $\mathrm{M} \phi$ and induce the gene expression of $I L-10$ and TGF- $\beta$ in vitro, suggesting that these nanovesicles directly interact with the innate immune cells. Several investigations have shown that EVs entered host cells via receptor-mediated pathways or lipid rafts [45, 53]. For $\mathrm{M} \phi$, the uptake of EVs may also be through random phagocytosis, and the underlying mechanism requires to be further explored. Besides, it is well-known that $M \phi$ is important participant in inflammatory responses, which produce multiple pro-inflammatory cytokines such as TNF- $\alpha$, IL-1 $\beta$ and IL-6 [54]. After challenge with LPS,
$\mathrm{M} \phi$ releases large quantities of these pro-inflammatory cytokines to promote inflammatory responses. In the model of LPS-activated $M \phi$ used in this study, we observed that LrEVs could effectively suppress the gene expression of TNF- $\alpha, I L-1 \beta$ and $I L-6$. Meanwhile, LrEVs also improved the gene expression of $I L-10$ and TGF- $\beta$. IL-10 and TGF- $\beta$ mainly mediate the negative regulation of inflammatory responses by suppressing the production of pro-inflammatory cytokines [55, 56]. Similar suppression of pro-inflammatory cytokines and activation of anti-inflammatory cytokines induced by $L$. paracasei-derived EVs have also been observed with LPS-challenged human colon cancer cells [15]. Furthermore, several studies have demonstrated that probiotics-derived EVs controlled inflammatory responses by modulating the cytokine production in the host's immune cells [31, 42]. Collectively, these findings support the suggestion that LrEVs can directly control the increased immune responses in inflammatory cells by modulating cytokine gene expression.

Previous studies have shown that $L$. reuteri has the ability to induce the development of effector $\mathrm{T}$ cells and forkhead box $\mathrm{P3}^{+}$(Foxp3 $^{+}$) Tregs in the mammalian gut $[21,22]$. Shen et al. revealed that gut commensal $B$. fragilis-derived EVs could induce the production of IL-10 in mouse DCs, which in turn promotes the development of Tregs [42]. Similarly, we found that LrEVs could elicit $I L-10$ gene expression in the cultured chicken $\mathrm{M} \phi$ alone, and the gene expression of $I L-10$ and TGF- $\beta$ in splenic lymphocytes were also up-regulated when they were cocultured with LrEVs-stimulated $\mathrm{M} \phi$. These findings indicate that LrEVs can induce anti-inflammatory responses in splenic lymphocytes through the activation of $\mathrm{M} \phi$. Moreover, IFN- $\gamma$ and IL-17 are representative Th1 and Th17 cytokines, respectively, which are mainly involved in the inflammatory responses [57]. Th1 and Th17-mediated inflammatory responses can be dampened by the Treg-derived IL-10 [57, 58]. The gene expression of IFN- $\gamma$ and $I L-17$ in splenic lymphocytes were down-regulated in this study under the cocultured condition with LrEVs-pretreated $M \phi$, implying that LrEVs can also inhibit the pro-inflammatory responses in splenic lymphocytes through the activation of $M \phi$. Furthermore, Foxp $3^{+}$Tregs play a curial role in inhibiting inflammatory responses in the intestine by secreting the anti-inflammatory cytokines IL-10 and TGF- $\beta$ [59]. Mammalian Foxp3 ${ }^{+}$Tregs can exert the immunosuppressive effect on effective $\mathrm{T}$ cells by increasing the gene expression of CTLA-4 [60]. The presence of $L A G-3$ in mammalian Tregs can inhibit the maturation of DCs, thus inducing the Treg-mediated suppression [61]. According to the results of splenic lymphocyte sub-population analysis with flow cytometer by Shanmugasundaram et al., the gene expression of CTLA-4 and LAG-3 can be 
served as markers for the development of chicken $\mathrm{CD} 44^{+} \mathrm{CD} 25^{+}$cells [62]. Although no Foxp3 ortholog genes have been identified in chickens to date, $\mathrm{CD} 4{ }^{+} \mathrm{CD} 25^{+}$cells from chicken spleen tissue have been shown to have the immunosuppressive and cytokine-producing properties of mammalian Foxp ${ }^{+}$Tregs [62]. The gene expression of $C D 25, C T L A-4$ and $L A G-3$ in splenic lymphocytes were enhanced in this study after coculture with LrEVs-pretreated $\mathrm{M} \phi$, implying that $\mathrm{CD} 4^{+} \mathrm{CD} 25^{+}$cells may participate in the LrEVs-mediated immunomodulation. However, whether $I L-$ 10 activated by LrEVs in $\mathrm{M} \phi$ is involved in the immunomodulatory activities of LrEVs-stimulated $\mathrm{M} \phi$ and exactly which lymphocyte subtypes are involved in the LrEVsmediated immunomodulation remains to be further studied.

Although the LrEVs-mediated immunomodulation was verified in this study, it is challenging to determine which specific molecules play the most important role. Among the compositions of bacterial EVs, proteins account for the largest proportion and mediate many functions of EVs [1]. Previously, it has been illustrated that certain proteins isolated from the probiotic-derived EVs exert a similar effect of the intact EVs. B. longum KACC 91563-derived EVs contain a protein ESBP that can induce the beneficial effect of the bacterial EVs [45]. EVs derived from L. casei BL23 carry several proteins associated with the probiotic effect of the bacteria, such as $\mathrm{p} 40$ and p75 [12]. In the present study, we characterized several proteins that are potentially associated with immune regulation in LrEVs, such as glucosyltransferase, serine protease, $60 \mathrm{kDa}$ chaperonin, elongation factor $\mathrm{Tu}$ and inositol polyphosphate phosphatase 1 . Bacterial glucosyltransferase may glycosylate the host target proteins, thus providing glycan decorations that can activate the DCSIGN signaling in DCs and induce the production of IL10 [63, 64]. Serine protease secreted by $L$. paracase has shown to be able to inhibit inflammatory responses by selectively targeting pro-inflammatory chemokines [65]. Bacterial $60 \mathrm{kDa}$ chaperonin, an heat shock protein analogue, may induce the development of $\mathrm{CD} 4{ }^{+} \mathrm{CD} 25^{+}$ Tregs and mediate TLR-2-dependent immunoregulation

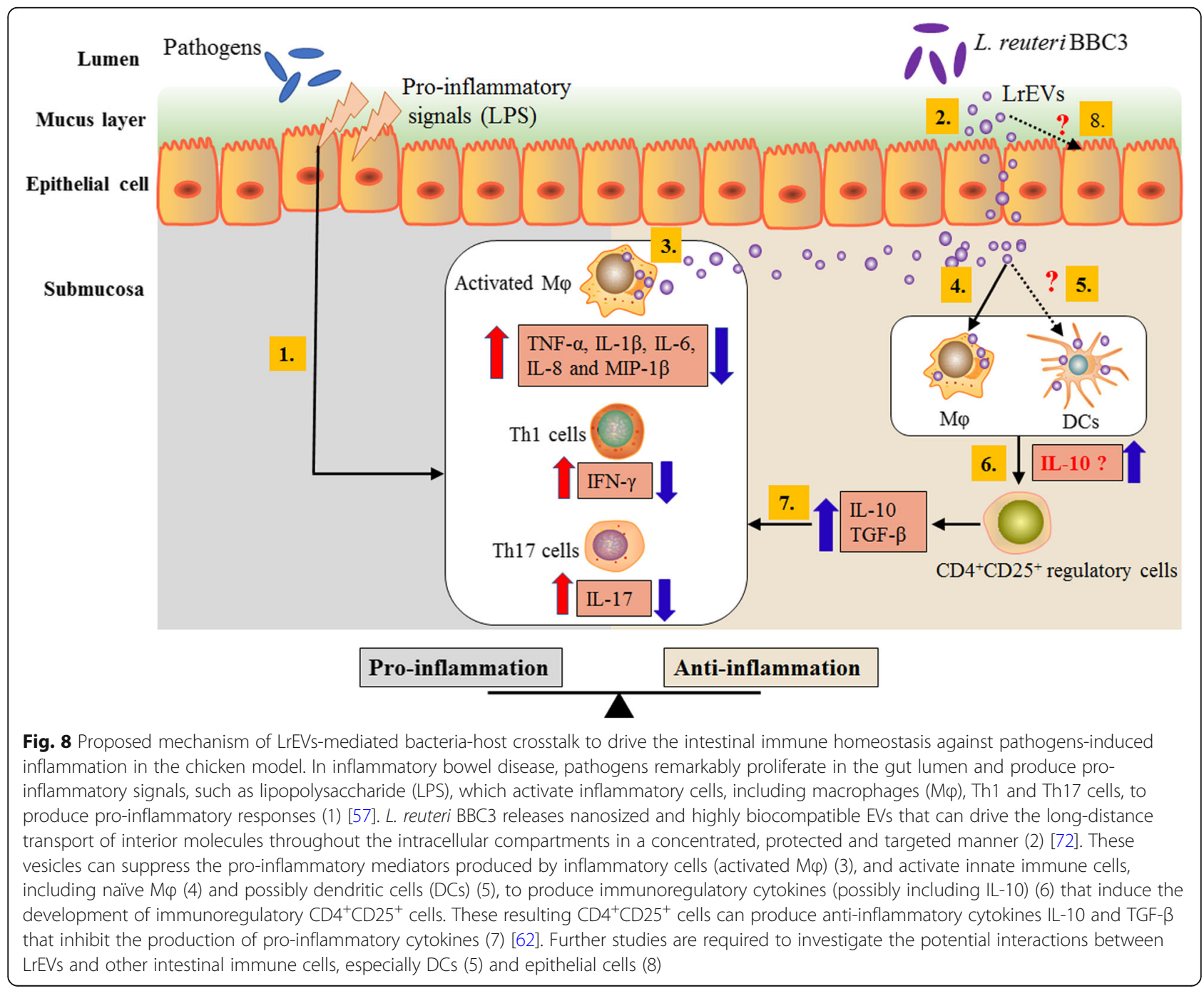


[66, 67]. Elongation factor $\mathrm{Tu}$ and inositol polyphosphate phosphatase 1 have also been observed to be involved in the intercellular signaling [68, 69]. Additionally, nucleic acid molecules derived from probiotics have also been proven to exert suppressive activity against inflammatory responses. Probiotics- and commensal bacteria-derived CpG DNA can mediate anti-inflammatory responses via Toll-like receptor 9 signaling [36, 70]. L. gasseri-derived RNA can inhibit inflammatory responses through a MyD88-dependent signaling pathway [71]. The study presented here confirmed that proteins and nucleic acids present in the LrEVs are essential for the LrEVs-mediated immunomodulation. Further studies will aim to explore the exact molecules involved in the immunoregulatory effect of LrEVs.

Due to the unique nano-scale structure of the lipid membrane-encapsulated vesicles, EVs can drive the longdistance transport of interior molecules throughout the intracellular compartments in a concentrated, protected and targeted manner [72]. According to the available results, we present a possible mechanism of LrEVs-mediated intestinal immune homeostasis against LPS-induced inflammation in the gut of the chicken model (Fig. 8). LrEVs are transported through intestinal epithelial cells, then suppress inflammatory responses in the activated $\mathrm{M} \phi$ and effector T cells, and promote the development of immunoregulatory $\mathrm{CD} 4{ }^{+} \mathrm{CD} 25^{+}$cells and the induction of anti-inflammatory cytokines. Further studies are needed to investigate the potential interactions between the LrEVs and other intestinal immune cells, especially epithelial cells and DCs.

\section{Conclusion}

In summary, we revealed that treatment of broiler chicks with LrEVs recapitulated the suppression of $L$. reuteri $\mathrm{BBC} 3$ on the LPS-induced intestinal injury and inflammation by modulating the gene expression of cytokines. We confirmed that LrEVs could mediate immune responses in LPS-activated $M \phi$ and splenic lymphocytes cocultured with $\mathrm{M} \phi$ in vitro. Moreover, we demonstrated that vesicular proteins and nucleic acids were required for LrEVsmediated bacteria-host interactions. To the best of our knowledge, this study is the first to reveal that the EVs secreted by $L$. reuteri played an important role in immune regulation and disease protection in the gut of the chicken model.

\section{Supplementary Information}

The online version contains supplementary material available at https://doi. org/10.1186/s40104-020-00532-4.

Additional file 1: Table S1. Primers used for quantitative real-time PCR in this study.

Additional file 2: Table S2. A total of 92 overlapping proteins

identified in triplicate biological samples of LrEVs.

\section{Abbreviations}

MVs: Membrane vesicles; EVs: Extracellular vesicles; OMVs: Outer membrane vesicles; TNF-a: Tumour necrosis factor a; IL: Interleukin; DCs: Dendritic cells; LPS: Lipopolysaccharide; M $\varphi$ : Macrophages; Tregs: Regulatory T cells; LrEVs: L. reuteri-derived EVs; PBS: Phosphate buffer saline; NTA: Nanoparticle tracking analysis; LC-MS/MS: Tandem mass spectrometry coupled online to the UPLC; GO: Gene Ontology; ADWG: Average daily weight gain; ADFI: Average daily feed intake; F/G: Feed gain ratio; Dil: Dialkylcarbocyanine iodide; DAPI: 4', 6Diamidino-2-phenylindole; FBS: Fetal bovine serum; MPO: Myeloperoxidase; qRT-PCR: Quantitative real-time PCR; SEM: Standard error of the mean; MIP: Macrophage inflammatory proteins; APCs: Antigen-presenting cells; Th: T helper; Foxp3: Forkhead box P3; CTLA-4: T-lymphocyte antigen 4; LAG3: Lymphocyte activation gene 3

\section{Acknowledgments}

The authors thank the State Key Laboratory of Crop Stress Biology for Arid Areas for providing the ultracentrifugation and electron microscopy analyses. The authors also thank Hangzhou PTM Biolabs for providing MS/MS data analysis.

\section{Authors' contributions}

RJH, YPG and MMY designed the study; RJH and MMY mainly wrote and revised the manuscript; YPG, XJY, JHY and MMY reviewed the manuscript; $\mathrm{RJH}, \mathrm{HL}, \mathrm{MMW}, \mathrm{YZZ}$ and HJL conducted the experiments and determination of parameters; YZZ, MMW, HJL and YNM contributed to animal feeding and data analyses. All authors read and approved the final manuscript.

\section{Funding}

This study was supported by the National Natural Science Foundation of China (No. 31372343 and 31672437).

\section{Availability of data and materials}

All data generated or analyzed during this study are included in this published article and its additional file.

\section{Ethics approval and consent to participate}

Experimental procedures and animal use were approved by the Northwest A\&F University Animal Care and Use Committee (Yangling, Shaanxi, China).

Consent for publication

Not applicable.

\section{Competing interests}

We declare that we have no competing interests.

Received: 17 June 2020 Accepted: 20 November 2020

Published online: 17 February 2021

\section{References}

1. Brown L, Wolf JM, Prados-Rosales R, Casadevall A. Through the wall: extracellular vesicles in gram-positive bacteria, mycobacteria and fungi. Nat Rev Microbiol. 2015;13(10):620-30.

2. Yáñez-Mó M, Siljander PR-M, Andreu Z, Zavec AB, Borràs FE, Buzas El, et al. Biological properties of extracellular vesicles and their physiological functions. J Extracell Vesicles. 2015:4(1):27066.

3. Kim JH, Lee J, Park J, Gho YS. Gram-negative and gram-positive bacterial extracellular vesicles. Semin Cell Dev Biol. 2015;40:97-104.

4. Schwechheimer C, Kuehn MJ. Outer-membrane vesicles from gram-negative bacteria: biogenesis and functions. Nat Rev Microbiol. 2015;13(10):605-19.

5. Acevedo R, Fernandez S, Zayas C, Acosta A, Elena Sarmiento M, Ferro VA, et al. Bacterial outer membrane vesicles and vaccine applications. Front Immunol. 2014;5:121.

6. van der Pol L, Stork M, van der Ley P. Outer membrane vesicles as platform vaccine technology. Biotechnol J. 2015;10(11):1689-706.

7. Lee EY, Choi DY, Kim DK, Kim JW, Park JO, Kim S, et al. Gram-positive bacteria produce membrane vesicles: proteomics-based characterization of Staphylococcus aureus-derived membrane vesicles. Proteomics. 2009;9(24): 5425-36.

8. Rivera J, Cordero RJB, Nakouzi AS, Frases S, Nicola A, Casadevall A. Bacillus anthracis produces membrane-derived vesicles containing biologically active toxins. P Natl Acad Sci USA. 2010;107(44):19002-7. 
9. Olaya-Abril A, Prados-Rosales R, McConnell MJ, Martin-Pena R, GonzalezReyes JA, Jimenez-Munguia I, et al. Characterization of protective extracellular membrane-derived vesicles produced by Streptococcus pneumoniae. J Proteome. 2014;106:46-60.

10. Brown L, Kessler A, Cabezas-Sanchez P, Luque-Garcia JL, Casadevall A. Extracellular vesicles produced by the gram-positive bacterium Bacillus subtilis are disrupted by the lipopeptide surfactin. Mol Microbiol. 2014;93(1): 183-98.

11. Jiang $Y$, Kong $Q$, Roland KL, Curtiss R III. Membrane vesicles of Clostridium perfringens type a strains induce innate and adaptive immunity. Int J Med Microbiol. 2014;304(3-4):431-43.

12. Rubio APD, Martinez JH, Casillas DCM, Leskow FC, Piuri M, Perez OE. Lactobacillus casei BL23 produces microvesicles carrying proteins that have been associated with its probiotic effect. Front Microbiol. 2017:8:1783.

13. Li M, Lee K, Hsu M, Nau G, Mylonakis E, Ramratnam B. Lactobacillus-derived extracellular vesicles enhance host immune responses against vancomycinresistant enterococci. BMC Microbiol. 2017;17(1):66.

14. Dean SN, Leary DH, Sullivan CJ, Oh E, Walper SA. Isolation and characterization of Lactobacillus-derived membrane vesicles. Sci Rep-Uk. 2019;9(1):1-11.

15. Choi JH, Moon CM, Shin T-S, Kim EK, McDowell A, Jo M-K, et al. Lactobacillus paracasei-derived extracellular vesicles attenuate the intestinal inflammatory response by augmenting the endoplasmic reticulum stress pathway. Exp Mol Med. 2020;52(3):423-37.

16. Grande R, Celia C, Mincione G, Stringaro A, Di Marzio L, Colone M, et al. Detection and physicochemical characterization of membrane vesicles (MVs) of Lactobacillus reuteri DSM 17938. Front Microbiol. 2017:8:1040.

17. Mu Q, Tavella VJ, Luo XM. Role of Lactobacillus reuteri in human health and diseases. Front Microbiol. 2018;9:757.

18. Walter J, Britton RA, Roos S. Host-microbial symbiosis in the vertebrate gastrointestinal tract and the Lactobacillus reuteri paradigm. P Natl Acad Sci USA. 2011:108:4645-52.

19. Christensen HR, Frøkiær H, Pestka JJ. Lactobacilli differentially modulate expression of cytokines and maturation surface markers in murine dendritic cells. J Immunol. 2002;168(1):171-8.

20. Lin YP, Thibodeaux CH, Peña JA, Ferry GD, Versalovic J. Probiotic Lactobacillus reuteri suppress proinflammatory cytokines via c-Jun. Inflamm Bowel Dis. 2008:14(8):1068-83.

21. Liu YY, Tran DQ, Fatheree NY, Rhoads JM. Lactobacillus reuteri DSM 17938 differentially modulates effector memory $T$ cells and Foxp3 ${ }^{+}$regulatory $T$ cells in a mouse model of necrotizing enterocolitis. Am J Physiol-Gastr $\mathrm{L}$. 2014;307(2):G177-86.

22. He B, Hoang TK, Wang T, Ferris M, Taylor CM, Tian X, et al. Resetting microbiota by Lactobacillus reuteri inhibits $T$ reg deficiency-induced autoimmunity via adenosine $A_{2 A}$ receptors. J Exp Med. 2016;214(1):107-23.

23. Hilmi HTA, Surakka A, Apajalahti J, Saris PE. Identification of the most abundant Lactobacillus species in the crop of 1- and 5-week-old broiler chickens. Appl Environ Microbiol. 2007;73(24):7867-73.

24. Mappley L, Tchórzewska MA, Nunez A, Woodward MJ, Bramley PM, La Ragione RM. Oral treatment of chickens with Lactobacillus reuteri LM1 reduces Brachyspira pilosicoli-induced pathology. J Med Microbiol. 2013; 62(2):287-96.

25. Nakphaichit M, Sobanbua S, Siemuang S, Vongsangnak W, Nakayama J, Nitisinprasert S. Protective effect of Lactobacillus reuteri KUB-AC5 against Salmonella Enteritidis challenge in chickens. Benef Microbes. 2019;10(1):43-54.

26. Prados-Rosales R, Brown L, Casadevall A, Montalvo-Quirós S, Luque-Garcia $J$. Isolation and identification of membrane vesicle-associated proteins in gram-positive bacteria and mycobacteria. MethodsX. 2014;1:124-9.

27. Hu R, Li J, Zhao Y, Lin H, Liang L, Wang M, et al. Exploiting bacterial outer membrane vesicles as a cross-protective vaccine candidate against avian pathogenic Escherichia coli (APEC). Microb Cell Fact. 2020;19(1):119.

28. Lee WH, Choi HI, Hong SW, Kim KS, Gho YS, Jeon SG. Vaccination with Klebsiella pneumoniae-derived extracellular vesicles protects against bacteriainduced lethality via both humoral and cellular immunity. Exp Mol Med. 2015;47(9):e183.

29. Chen $Y$, Zhang H, Cheng Y, Li Y, Wen C, Zhou Y. Dietary L-threonine supplementation attenuates lipopolysaccharide-induced inflammatory responses and intestinal barrier damage of broiler chickens at an early age. Brit J Nutr. 2018;119(11):1254-62.

30. Beug H, von Kirchbach A, Döderlein G, Conscience J-F, Graf T. Chicken hematopoietic cells transformed by seven strains of defective avian leukemia viruses display three distinct phenotypes of differentiation. Cell. 1979;18(2):375-90.

31. Forsberg MM, Björkander S, Pang $Y$, Lundqvist $L, N$ di $M$, Ott $M$, et al. Extracellular membrane vesicles from Lactobacilli dampen IFN- $\gamma$ responses in a monocyte-dependent manner. Sci Rep-Uk. 2019;9(1):1-13.

32. Zhang Q, Chen X, Eicher S, Ajuwon K, Applegate T. Effect of threonine on secretory immune system using a chicken intestinal ex vivo model with lipopolysaccharide challenge. Poultry Sci. 2017;96(9):3043-51.

33. Livak KJ, Schmittgen TD. Analysis of relative gene expression data using real-time quantitative PCR and the $2^{-\Delta \Delta C t}$ method. Methods. 2001;25(4): 402-8.

34. Strober W. Trypan blue exclusion test of cell viability. Current Protocols in Immunology. 1997;21(1):A.3B.1-2.

35. Jiang LL, Shen YY, Guo DF, Yang DY, Liu JJ, Fei XF, et al. EpCAM-dependent extracellular vesicles from intestinal epithelial cells maintain intestinal tract immune balance. Nat Commun. 2016;7(1):1-16.

36. Walker WA. Mechanisms of action of probiotics. Clin Infect Dis. 2008; 46(Suppl 2):S87-91.

37. Thaiss CA, Zmora N, Levy M, Elinav E. The microbiome and innate immunity. Nature. 2016;535(7610):65-74

38. Macia L, Nanan R, Hosseini-Beheshti E, Grau GE. Host- and microbiotaderived extracellular vesicles, immune function, and disease development. Int J Mol Sci. 2020;21(1):107.

39. Kaparakis-Liaskos M, Ferrero RL. Immune modulation by bacterial outer membrane vesicles. Nat Rev Immunol. 2015;15(6):375-87.

40. Kesty NC, Mason KM, Reedy M, Miller SE, Kuehn MJ. Enterotoxigenic Escherichia coli vesicles target toxin delivery into mammalian cells. EMBO J. 2004;23(23):4538-49.

41. Rueter C, Bielaszewska M. Secretion and delivery of intestinal pathogenic Escherichia coli virulence factors via outer membrane vesicles. Front Cell Infect Mi. 2020;10:91

42. Shen Y, Torchia MLG, Lawson GW, Karp CL, Ashwell JD, Mazmanian SK. Outer membrane vesicles of a human commensal mediate immune regulation and disease protection. Cell Host Microbe. 2012;12(4):509-20.

43. Kang CS, Ban M, Choi EJ, Moon HG, Jeon JS, Kim DK, et al. Extracellular vesicles derived from gut microbiota, especially Akkermansia muciniphila, protect the progression of dextran sulfate sodium-induced colitis. PLoS One. 2013:8(10):e76520.

44. Fábrega MJ, Aguilera L, Giménez R, Varela E, Cañas MA, Antolin M, et al. Activation of immune and defense responses in the intestinal mucosa by outer membrane vesicles of commensal and probiotic Escherichia coli strains. Front Microbiol. 2016;7:705.

45. Kim J-H, Jeun E-J, Hong C-P, Kim S-H, Jang MS, Lee E-J, et al. Extracellular vesicle-derived protein from Bifidobacterium longum alleviates food allergy through mast cell suppression, J Allergy Clin Immun. 2016;137(2):507-16 e8.

46. Guijarro-Muñoz I, Compte M, Álvarez-Cienfuegos A, Álvarez-Vallina L, Sanz L. Lipopolysaccharide activates toll-like receptor 4 (TLR4)-mediated NF-KB signaling pathway and proinflammatory response in human pericytes. J Biol Chem. 2014:289(4):2457-68.

47. Liu S, Song M, Yun W, Lee J, Kim H, Cho J. Effect of carvacrol essential oils on immune response and inflammation-related genes expression in broilers challenged by lipopolysaccharide. Poultry Sci. 2019:98(5):2026-33.

48. Hu X, Guo Y, Li J, Yan G, Bun S, Huang B. Effects of an early lipopolysaccharide challenge on growth and small intestinal structure and function of broiler chickens. Can J Anim Sci. 2011;91(3):379-84.

49. Li R, Song Z, Zhao J, Huo D, Fan Z, Hou D-X, et al. Dietary L-theanine alleviated lipopolysaccharide-induced immunological stress in yellowfeathered broilers. Anim Nutr. 2018:4(3):265-72.

50. Yang L, Liu G, Zhu X, Luo Y, Shang Y, Gu X-L. The anti-inflammatory and antioxidant effects of leonurine hydrochloride after lipopolysaccharide challenge in broiler chicks. Poultry Sci. 2019;98(4):1648-57.

51. Li R, Li J, Zhang S, Mi Y, Zhang C. Attenuating effect of melatonin on lipopolysaccharide-induced chicken small intestine inflammation. Poultry Sci. 2018;97(7):2295-302

52. Abe M, Hiura K, Wilde J, Moriyama K, Hashimoto T, Ozaki S, et al. Role for macrophage inflammatory protein (MIP)- $1 a$ and MIP-1 $\beta$ in the development of osteolytic lesions in multiple myeloma. Blood. 2002:100(6):2195-202.

53. Kaparakis M, Turnbull L, Carneiro L, Firth S, Coleman HA, Parkington HC, et al. Bacterial membrane vesicles deliver peptidoglycan to NOD1 in epithelial cells. Cell Microbiol. 2010;12(3):372-85. 
54. Murray PJ, Wynn TA. Protective and pathogenic functions of macrophage subsets. Nat Rev Immunol. 2011;11(11):723-37.

55. Letterio JJ, Roberts AB. Regulation of immune responses by TGF- $\beta$. Annu Rev Immunol. 1998;16(1):137-61.

56. Fiorentino DF, Zlotnik A, Mosmann T, Howard M, O'garra A. IL-10 inhibits cytokine production by activated macrophages. J Immunol. 1991;147(11): 3815-22.

57. Arrieta M-C, Finlay BB. The commensal microbiota drives immune homeostasis. Front Immunol. 2012;3:33.

58. Weaver $C T$, Harrington LE, Mangan PR, Gavrieli M, Murphy KM. Th17: an effector CD4 T cell lineage with regulatory T cell ties. Immunity. 2006;24(6): 677-88.

59. Vignali DAA, Collison LW, Workman CJ. How regulatory T cells work. Nat Rev Immunol. 2008;8(7):523-32.

60. Wing K, Onishi Y, Prieto-Martin P, Yamaguchi T, Miyara M, Fehervari Z, et al. CTLA-4 control over Foxp3 ${ }^{+}$regulatory T cell function. Science. 2008; 322(5899):271-5.

61. Huang C-T, Workman CJ, Flies D, Pan X, Marson AL, Zhou G, et al. Role of LAG-3 in regulatory T cells. Immunity. 2004;21(4):503-13.

62. Shanmugasundaram R, Selvaraj RK. Regulatory $T$ cell properties of chicken $\mathrm{CD4}^{+}$CD25 ${ }^{+}$cells. J Immunol. 2011;186(4):1997-2002.

63. van Baarlen P, Wells JM, Kleerebezem M. Regulation of intestina homeostasis and immunity with probiotic Lactobacilli. Trends Immunol. 2013;34(5):208-15.

64. Gao L, Song QT, Liang H, Zhu YT, Wei TT, Dong N, et al. Legionella effector SetA as a general O-glucosyltransferase for eukaryotic proteins. Nat Chem Biol. 2019;15(3):213-6.

65. von Schillde MA, Hormannsperger G, Weiher M, Alpert CA, Hahne H, Bauerl C, et al. Lactocepin secreted by Lactobacillus exerts anti-inflammatory effects by selectively degrading proinflammatory chemokines. Cell Host Microbe. 2012;11(4):387-96.

66. Wang $X$, Zhou S, Chi Y, Wen X, Hoellwarth J, He L, et al. CD4 ${ }^{+} \mathrm{CD} 25^{+}$Treg induction by an HSP60-derived peptide SJMHE1 from Schistosoma japonicum is TLR2 dependent. Eur J Immunol. 2009;39(11):3052-65.

67. Al-Nedawi K, Mian MF, Hossain N, Karimi K, Mao YK, Forsythe P, et al. Gut commensal microvesicles reproduce parent bacterial signals to host immune and enteric nervous systems. FASEB J. 2015:29(2):684-95.

68. Granato D, Bergonzelli GE, Pridmore RD, Marvin L, Rouvet M, CorthesyTheulaz IE. Cell surface-associated elongation factor Tu mediates the attachment of Lactobacillus johnsonii NCC533 (La1) to human intestinal cells and mucins. Infect Immun. 2004;72(4):2160-9.

69. Stentz R, Osborne S, Horn N, Li AWH, Hautefort I, Bongaerts R, et al. A bacterial homolog of a eukaryotic inositol phosphate signaling enzyme mediates cross-kingdom dialog in the mammalian gut. Cell Rep. 2014;6(4): 646-56

70. Rachmilewitz D, Katakura K, Karmeli F, Hayashi T, Reinus C, Rudensky B, et al. Toll-like receptor 9 signaling mediates the anti-inflammatory effects of probiotics in murine experimental colitis. Gastroenterology. 2004;126(2):520-8.

71. Yoshida A, Yamada K, Yamazaki Y, Sashihara T, Ikegami S, Shimizu M, et al. Lactobacillus gasseri OLL2809 and its RNA suppress proliferation of CD4 ${ }^{+} T$ cells through a MyD88-dependent signaling pathway. Immunology. 2011; 133(4):442-51.

72. Kulp A, Kuehn MJ. Biological functions and biogenesis of secreted bacterial outer membrane vesicles. Annu Rev Microbiol. 2010;64:163-84.

Ready to submit your research? Choose BMC and benefit from:

- fast, convenient online submission

- thorough peer review by experienced researchers in your field

- rapid publication on acceptance

- support for research data, including large and complex data types

- gold Open Access which fosters wider collaboration and increased citations

- maximum visibility for your research: over $100 \mathrm{M}$ website views per year

At $\mathrm{BMC}$, research is always in progress.

Learn more biomedcentral.com/submissions 